\title{
Parabolische Regularisierung einer hyperbolischen Itogleichung
}

\author{
W. GRECKSCH
}

Die Lösung der ersten Anfangs-Randwert-Aufgabe für eine stochastische hyperbolische Gleichung wird mit einer Methode der parabolischen Regularisierung approximiert. Auf der Grundlage dieses Konzeptes werden ein Maximumprinzip und die Existenz e-optimaler Steuerungen für eine Steueraufgabe einer speziellen hyperbolischen stochastischen Gleichung untersucht.

Решение первой начально-краевой задачи для некоторого стохастического гиперболического уравнения аптроксимировано методом параболической регуляризации. На основе әтой концепции исследованы принцип максимума и существование $\varepsilon$-оптимальных управленй для задачи оптимального управления одного гиперболического стохастического уравнения.

The solution of the first boundary value problem of a random hyperbolic equation is approximated by a method of the parabolic regularization. Considered an application of this method to the development of a maximum principle and to the existence of the $\dot{\varepsilon}$-optimal controls of an optimal control problem of a random hyperbolic equation.

\section{Einleitung}

Mit der Theorie der monotonen Operatoren (s. z. B. [14]) und der stochastischen Integration unbeschränkter Operatoren [12] sind wesentliche Hilfsmittel zur Untersuchung von Itogleichungen, die partielle Ableitungen enthalten, bereitgestellt. Fine wichtige Frage dabei ist die Approximation der Lösungen solcher Gleichungen. In [8] wird für eine allgemeine nichtlineare parabolische Itogleichung die Ritz-GalerkinMethode angewendet. Unter bestimmten Voraussetzungen ist die Anwendung der Halbgruppentheorie möglich [3]. Lösungen hyperbolischer Itogleichungen werden z. B. in [6] und [13] mit der Ritz-Galerkin-Methode approximiert. Für eine spezielle lineare hyperbolische Itogleichung wird in [5] eine Fourierapproximation angewendet.

In vorliegender Arbeit werden Lösungen linearer hyperbolischer Itogleichungen durch Anwendung einer parabolischen Regularisierung approximiert. Fiir eine Steueraufgabe mit einer linearen hy perbolischen Itogleichung wird auf der Grundlage dieses Konzeptes ein Maximumprinzip hergeleitet und die Frage nach $\varepsilon$-optimalen Steuerungen untersucht.

Die Methode der parabolischen Regularisierung ist im deterministischen Fall wohlbekannt. $[10,11]$.

\section{Eine parabolische Itogleichung}

Zun̈ächst werden einige Bezeichnungen eingeführt:

$(\Omega, \mathfrak{F}, P) \quad$ Vollständiger Wahrscheinlichkeitsraum.

$E(\cdot)$

$[0, T]$

Erwartungswertoperator.

Beschränktes abgeschlossenes Intervall. 
(F) Familie von $\sigma$-Algebren aus $\mathfrak{j}$ mit $\mathfrak{F}_{s} \subseteq \mathfrak{F}_{i}$ für $s \leqq t$.

$K, H, M$ 、. Separable Hilberträume mit den Skalarprodukten $(\cdot, \cdot)_{K},(\cdot, \cdot)_{H}$ und $(\cdot, \cdot)_{M}$. Die dualen Räume $K^{*}, H^{*}, M^{*}$ werden mit $K, H$ und $M$ identifiziert.

$L(K, H) \quad$ Raum der linearen stetigen Abbildungen von $K$ in $H$.

$\mathfrak{B}_{Z}, \sigma$-Algebra der Borelıengen eines Banachraumes $Z$.

$\left(w(t), \mathfrak{F}_{t}\right) \quad K$-wertiger Wienerprozeß $\mathrm{mit}$ einem Kernoperator $Q$ als Kovarianzoperator.

$V, U$ 'Reflexive separable Banachräume.

$V^{*}, U^{*} \quad Z u V$ und $U$ duale Räume.

$\left\langle v^{*}, v\right\rangle_{V} \quad$ Wert des linearen stetigen Funktionales $v^{*} \in V^{*}$ an der Stelle $v \in V$. Analog ist $\left\langle u^{*}, u\right\rangle_{U}$ zui verstehen.

$V \subset H \subset V^{*}$ Evolutionstripel (s. [14]).

$U \subset C \subset U^{*}$ Evolutionstripel.

$\mathscr{L}_{Q}, \quad$ Raum der linearen Operatoren $\Phi\left|Q^{1 / 2} K\right| \rightarrow H$, so da $\$ \Phi Q^{1 / 2}$ HilbertSchmidt-Operatoren von $K$ in $H$ sind. Versehen mit dem Skalarprodukt $\left(\Phi, \Psi_{Q}:=\operatorname{tr}\left(\Phi Q^{1 / 2}\right) \cdot\left(\Psi^{\prime} Q^{1 / 2}\right)\right.$ ist $\mathscr{L}_{Q}$ ein Hilbertraum.

$\mathscr{B}_{Q} \cdot \sigma$-Algebra der Borelmengen aus $\mathscr{L}_{G}$.

$\mathscr{L}_{Z}{ }^{2} \quad$ Iuebesguescher Raum $L_{Z}{ }^{2}\left([0, T] \times \Omega, \mathfrak{B}_{[0, T]} \times \mathfrak{F}, d t \otimes d P\right)(Z-$ refle$\tilde{\mathscr{\mathscr { P }}} \quad$ xiver separabler Banachraum).

$\tilde{\mathscr{L}}_{Z}{ }^{2} \quad$ Teilraum der $\left(\mathscr{F}_{t}, \mathfrak{B}_{Z}\right)$-melbaren Elemente aus $\mathscr{L}_{Z}{ }^{2}$.

Fïr alle $(v, t, \omega) \in U \times\left[0, T^{i}\right] \times \Omega$ seien $A(v, t, \omega) \in U^{*}$ und $B(v, t, \omega) \in \mathscr{L}_{u}$. Für jedes $v \in U$ seien die Funktionen $A(v, t, \omega)$ und $B(v, t, \omega)$ sowohl $\left(\mathfrak{B}_{[0, T]} \times \mathfrak{F}, \mathfrak{B}_{U^{*}}\right)$ als auch $\left(\mathfrak{B}_{[0, T]} \times \mathfrak{F}, \mathfrak{B}_{Q}\right)$-meßbar. Es wird weiter vorausgesetzt, da $B$ für jedes $t \in[0, T]$ und $z \in U$ die Funktionen $A(v):=A(v, t, \omega)$ und $B(v):=B(v, t, \omega)$ bezüglich $\omega\left(\mathfrak{F}_{l}, \mathfrak{B}_{U^{*}}\right)$-meßbar und $\left(\mathfrak{F}_{l}, \mathfrak{B}_{Q}\right)$-meßbar sind. Schließlich bezeichnen $(z(t))$ ein $H$-wertiges quadratisch integrierbares Martingal, $X_{0}$ eine $\left(\mathfrak{F}_{0}, \mathfrak{B}_{H}\right)$-mebbare Funktion und $f(t, \omega)$ eine $\left(\mathfrak{B}_{[0, T]} \times \mathfrak{F}, \mathfrak{B}^{1}\right)$-meßbare nichtnegative integrierbare Funktion, die für jedes $t \in[0, T]\left(\mathfrak{J}_{t}, \mathcal{B}^{1}\right)$-melßbar ist. Weiter seien $\left.p \in\right] 1, \infty[, q:=p /(p-1)$.

In [8] wird bewiesen

Satz 1:1: Es gebe positive Konstanten $k$ und $\alpha$, so $d u \beta$ für alle $v_{1}, v_{2}, \imath \in U$, $t \in[0, T], \omega \in \Omega$ folgende Bedingungen erfüllt sind:

$\left(\mathbf{A}_{1}\right)$ Die Funktion $\left\langle A\left(v_{1}+i v_{2}\right), v\right\rangle_{V}$ ist bezüglich $i \in \mathbf{R}^{1}$ stctig.

(A) $2\langle A v, v\rangle_{U}+\|B(v)\|_{Q}^{2}+\alpha\|v\|_{0}^{p} \leqq f+k\|v\|_{H}{ }^{2}$.

(A $\left.\mathrm{A}_{3}\right) 2\left\langle A\left(v_{1}\right)-A\left(v_{2}\right), v_{1}-v_{2}\right\rangle_{l}+\left\|B\left(v_{1}\right)-B\left(v_{2}\right)\right\|_{Q}^{2} \leqq k\left\|v_{1}-v_{2}\right\|_{H}{ }^{2}$.

$\left(\mathrm{A}_{4}\right)\|A(v)\|_{U^{*}} \leqq f^{1 / q}+k\|v\|_{l^{p \cdot 1}}$.

(A) $A_{5}\left\|X_{0}\right\|_{H}{ }^{2}<\infty$.

Dunn gibt es eine $H$-wertige, auf $[0, T] \times Q$ definierte Funktion $X(t):=X(t, \omega)$, - die für alle $t \in[0, T]\left(\mathfrak{F}_{\iota}, \mathfrak{B}_{H}\right)$-meßbur und für $\omega \in \Omega$ bezüglich $t \in[0, T]$ stetig in $H$ ist, so daß gilt:

1. $X(t, \omega) \in U$ für fast alle $(t, \omega) \in[0, I] \times \Omega$.

2. Es existiert ein $\Omega^{\prime} \subset \Omega$ mit $P\left(\Omega^{\prime}\right)=1$ und auf $\Omega^{\prime}$ gilt für alle' $t \in[0, T]$ die Itogleichung

$$
\left.X(t)=X_{0}+\int_{0}^{t} A(X(s), s,(s)) d s+\int_{0}^{t} B(X(s), s, \omega) w(d s)+z(t) \cdot{ }^{1}\right)
$$

1) Das stochastische Integral ist wie in [12] definiert. 
3. $-E \sup _{i}\|X(t)\|_{H^{2}}+E \int_{0}^{T}\|X(t)\|_{U}^{p} d t$

$$
\leqq \text { const }\left(E\left\|X_{0}\right\|_{H}^{2}+E \int_{0}^{\bar{T}} f(t) d t+E\|z(T)\|_{H^{2}}\right) \text {. }
$$

4. $(X(t))$ ist bis auf stochastische Modifikationen eindeutig bestimmt.

Bemerkung 1.2: Für die Gleichung (1.1) wird symbolisch geschrieben

$$
\left\{\begin{array}{l}
d X(t)=A(X(t), i, \omega) d t+B(X(t), t, \omega) w(d t)+z(d t), \\
X(0)=X_{0}
\end{array}\right.
$$

Bemerkiung 1.3: $(X(t))$ ist Lösung der Gleichung ${ }^{-}(1.1)$ im Sịne des Satzes 1.1 genau dann, wenn für alle $v \in Y^{\prime}\left(Y-\right.$ in $U$ dichte Menge beziiglich $\left.\|\cdot\|_{U}\right)$ gilt:

$$
\begin{aligned}
(v, X(t))_{H}=\left(v, X_{0}\right)_{H} & +\int_{0}^{t}\langle A(X(s), s, \omega), v\rangle_{v} d s \\
& +\int_{0}^{t}(v, B(X(s), s, \omega), w(d s))_{H}+(v, z(t))_{H} .
\end{aligned}
$$

Bemerkung 1.4: Die Bemerkung 1.3 zeigt, daß der Lösungsbegriff im Satz 1.1 eine Úbertragung des Lösungsbegriffes der verallgemeinerten Lösung einer deterṇinistischen parabolischen Gleichung [11] iiber einem Evolutionstripel $\dot{U} \subset H \subset U^{*}$ ist.

\section{Eine hyperbolische Itogleichung}

Es sei $\left(V, H, V^{*}\right)$ ein Evolutionstripel von Hilberträumen. Wir untersuchen die Itogleichung

$$
\begin{aligned}
& d y^{\prime}(t)+A \dot{y}(t) \dot{d} t \\
& =g(t) d t+B_{1}(t, \omega) y(t) w(d t)+B_{2}(t, \omega) y^{\prime}(t) w(d t)+z(d t)
\end{aligned}
$$

mit den Anfangsbedingungen $y(0)=y_{0}, y^{\prime}(0)=y_{1}^{\prime}\left(y_{0} \in V, y_{1} \in H\right)$. Dabei sind $(g(t)) \in \tilde{\mathscr{L}}_{H^{2}}, A\left|V \rightarrow V^{*}, B_{1}, B_{2}\right|[0, T] \times \Omega \times V \rightarrow \mathscr{L}_{Q}$. Für Funktionen $y \in L_{V^{2}}$ - $\times\left([0, T], B_{i 0, T]}, d t\right)$ bezeichne $y^{\prime}(t)$ die erste Ableitung im Sinne der Distributionen. Fïr die Abbildungen $A, B_{1}^{\prime}$ und $B_{2}$ wird vorausgesetzt:

$\left(V_{1}\right) A$ ist linear, und es gibt eine positive $Z a h l \delta$, so daß für alle $v \in \underline{V}$ gilt

$$
\langle A v ; v\rangle_{V} \geqq \delta\|v\|_{V^{2}} \text {. }
$$

$\left(V_{2}\right)$ Die Funktionen $B_{i}(t) \bar{v}:=B_{i}(t, \omega) \cdot v(i=1,2)$ sind bezüglich $v$ linear, und es gibt eine positive Konstante $h$, so da $\beta$ fiir alle $t \in[0, T], \omega \in \Omega$ und $v \in V$ gilt

$$
\left\|B_{1}(t) v\right\|_{Q^{2}} \leqq h\|v\|_{H^{2}}, \quad\left\|B_{2}(t) V\right\|_{Q^{2}} \leqq h\|v\|_{H}{ }^{2} .
$$

$\left(V_{3}\right)$ Fiir jedes $v \in V$ sind $B_{i}(t) v(i=1,2)$ als Funktionen ïber $[0, T] \times \Omega$ $\left(\mathfrak{B}_{[0, T)} \times \mathfrak{j}, \mathfrak{B}_{Q}\right)$-meßbar.

$\left(V_{4}\right)$ Fiir jedes $t \in[0, T]$ und $v \in V$ sind die Funktionen $B_{i}(l) v(i=1,2)$ iiber $\Omega\left(\Im_{1}, \mathfrak{b}_{Q}\right)$-meßbar. 
Bemerkung 2.1: Ein Beispiel für die Gleichung (2.1) über dem Evolutionstripel $\left(\stackrel{W}{W}_{2}^{1}(G), L^{2}(G), W_{2}^{-1}(G)\right)$ (Bezeichnung der-Sobolewräune wie in [8]) ist die Anfangswertaufgabe

$d\left[\frac{\partial y\left(t ; x_{1}, \ldots, x_{d}\right)}{\partial_{\ell}}\right]-\left[\sum_{j, k=1}^{d} \frac{\partial}{\partial x_{j}}\left(A_{j k}\left(x_{1}, \ldots, x_{d}^{\prime}\right) \frac{\partial y\left(t ; x_{1}, \ldots, x_{d}\right)}{\partial x_{k}}\right)+C\left(x_{1}, \ldots, x_{d}\right)\right] \cdot d i$

$=g\left(t ; x_{1}, \ldots, x_{d}\right) d t+y\left(t ; x_{1}, \ldots, x_{t}\right) \beta(d l), \quad y(0)=y_{0}(x)$,

$$
\left.\frac{\partial y\left(t ; x_{1}, \ldots, x_{d}\right)}{\partial t}\right|_{t=0}=y_{1}(x),\left.y\left(t ; x_{1}, \ldots, x_{d}\right)\right|_{x \in \partial G}=0
$$

nuit deterministischen Koeffizienten $A_{j k}(x), C(x)$. Jabei sind: $(\beta(t))$ ein reellwertiger Wienerproze $B, G$ ein beschränktes Gebiet im $\underset{T}{\mathbf{R}^{d}}, \partial G$ der Rand von $G, \cdot\left(g\left(t ; x_{1}, \ldots, x_{d}\right)\right)$ $\operatorname{ein} L^{2}(G)$-wertiger $\Im_{t}$-mel 3 barer Prozeß mit $E \int_{0}^{T} \int_{G}\left|g\left(t ; x_{1}, \ldots, x_{d}\right)\right|^{2} d x_{1} \ldots d x_{d} d t<\infty$, $A_{j k}(x), C(x)$ beschränkte imeßbare Funktionen nit $\sum_{j=1}^{d} A_{j k}\left(x_{1}, \ldots, x_{d}\right) z_{j} z_{k} \geq$ const. $\|z\|_{\mathbf{R}^{d}}^{2}$ fiir alle $x:=\left(x_{1}, \ldots, x_{d}\right) ; z:=\left(z_{1}, \ldots, z_{d}\right) \in \mathbf{R}^{d}$.

In [5] wird die Lösung der (Gleichung (2.4) mittels der Fourierapproximation bestimmt.

Bemerkung. 2.2: Eine Gleichung vom Typ (2.4), wobei in die linke Seite noch additiv $y^{\prime}(t) d t$ eingeht, $\beta(d t)$ rein additiv wirkt und die Koeffizienten $A_{j k}$ und $C$ noch von $t$ abhängen können, wird in [6] untersucht. Die Tösung wird durch ein Verfahren vom Galerkin-Typ approximiert.

Bemerkung 2.3: 'Tritt in der linken Seite der Gleichung (2.1) noch ein Term des Types $A_{1}\left(t, \omega, y^{\prime}(t)\right)$ auf, so ist.die Gleichung unter Monotonie- und Koerzitivitätsvoraussetzungen in [13] diskutiert worden.

Bemerkung 2.4: Im deterministischen Fall (d. h. $B_{1}(t) \equiv 0, B_{2}(t) \equiv 0, z(t) \equiv 0$, $g$ ist nicht von $\omega$ abhängig) kann eine. Lösung der Gleichung (2.1) durch eine parabolische Regularisierung approximiert werden.

Bemerkung 2.5: Die folgenden Aussagen sind auch anwendbar, wenn $B_{2}(t) \equiv 0$ und $B_{1}(t) \neq 0$ gilt.

Beispiel 2.6: Wir betrachten das Evolutionstripel $\left(\stackrel{\circ}{W}_{2}^{1}(G), L^{2}(G), W_{2}^{-1}(G)\right)$ mit $G=[0,1] \times[0,1] . A_{j k}, C$ und $g$ erfïilen mit $d=2$ die Voraussetzungen der Bemerkung 2.1:

$$
\begin{aligned}
& d\left[\frac{\partial y\left(t ; x_{1}, x_{2}\right)}{\partial t}\right] \\
& -\left\{\sum_{j, k=1}^{2} \frac{\partial}{\partial x_{j}}\left[A_{j k}\left(x_{1}, x_{2}\right) \frac{\partial y\left(t ; x_{1}, x_{2}\right)}{\partial x_{k}}\right]+C\left(x_{1}, x_{2}\right) y\left(t ; x_{1}, x_{2}\right)\right\} d t \\
& =g\left(t ; x_{1}, x_{2}\right) d t+y\left(t ; x_{1}, x_{2}\right) \beta(d t)+\left[\frac{\partial}{\partial x_{1}} y\left(t ; x_{1}, x_{2}\right)+\frac{\partial}{\partial x_{2}} y\left(t ; x_{1}, x_{2}\right)\right] \beta(d t), \\
& y\left(0 ; x_{1}, x_{2}\right)=y_{0}\left(x_{1}, x_{2}\right),\left.\frac{\partial y\left(t ; x_{1}, x_{2}\right)}{\partial t}\right|_{t=0}=y_{1}\left(x_{1}, x_{2}\right),\left.y\left(t ; x_{1}, x_{2}\right)\right|_{\left(x_{1}, x_{2}\right) \epsilon \partial G}=0 .
\end{aligned}
$$




\section{Parabolische Regularisierung}

Für.positive Parameter $\varepsilon$ wird die Jtogleichung

$$
\begin{aligned}
& d y_{\varepsilon}^{\prime}(t)+A y_{\varepsilon}(t) d t+\varepsilon A y_{\varepsilon}^{\prime}(t) d t \\
& =g(t) d t+B_{1}(t) y_{c}(t) w(d t)+B_{2}(t) y_{\varepsilon}^{\prime}(t) w(d t)+z(d t) \\
& \text { mit } y(0)=y_{0}, \quad y^{\prime}(0)=y_{1}
\end{aligned}
$$

betrachtet.

Satz 3.1: Für alle $\varepsilon>0$ gibt es einen $V \times V$-weriigen $\mathfrak{J}_{t}$-meßbaren Proze $\beta\left(y_{\varepsilon}(t)\right.$, $\left.y_{c}^{\prime}(t)\right)$, der in $V \times H$ bezuiglich $t \in[0, T]$ für alle $\omega \in \Omega$ stetig ist und auf einer Menge $\Omega_{0}$ mit $P\left(\Omega_{0}\right)=1$ für alle $t \in[0, T]$ der Gleichung (3.1) genügt. Dieser Proze $\beta$ ist bis auf stochustische Modifikationen einileutig bestimmt, und es gilt.

$$
E \sup _{t}\left(\left\|y_{\varepsilon}(t)\right\|_{v^{2}}+\left\|y_{\varepsilon}{ }^{\prime}(t)\right\|_{H^{2}}\right)+E \int_{0}^{T}\left(\left\|y_{\varepsilon}(t)\right\|_{V^{2}}+\left\|y_{\varepsilon}{ }^{\prime}(t)\right\|_{v^{2}}\right) d t \leqq \text { const }
$$

für $\varepsilon \in] 0,1]$.

Beweis: Hs werden die Produkträume $\mathfrak{B}:=V \times V, \mathfrak{E}:=V \times H$ und $\mathfrak{Y}^{\prime}:=V \times V^{*}$ eingefiihrt. Uber $\mathfrak{S}$ wird durch

$$
\lceil\varphi, \psi]:=\left\langle A \varphi_{0}, \psi_{0}\right\rangle_{V}+\left(\varphi_{1}, \psi_{1}\right)_{H} \quad \text { mit } \quad \varphi:=\left(\begin{array}{l}
\varphi_{0} \\
\varphi_{1}
\end{array}\right), \psi:=\left(\begin{array}{l}
\psi_{0} \\
\psi_{1}
\end{array}\right) \text {, }
$$

ein Skalarprodukt definiert,

$$
\left\langle\varphi^{*}, \varphi\right\rangle_{\mathfrak{B}}:=\left\langle A \varphi_{0}{ }^{*}, \varphi_{0}\right\rangle_{V}+\left\langle\varphi_{1}{ }^{*}, \varphi_{1}\right\rangle_{V} \text { mit } \varphi^{*}:=\left(\begin{array}{l}
\varphi_{0}{ }^{*} \\
\varphi_{1}{ }^{*}
\end{array}\right) \in \mathfrak{B}^{\prime}, \varphi:=\left(\begin{array}{l}
\varphi_{0} \\
\varphi_{\mathrm{J}}
\end{array}\right) \in \mathfrak{B}
$$

definiert auf $\mathfrak{B} \times \mathfrak{\mathfrak { B } ^ { \prime }}$ eine Bilinearform. Unter Zugrundelegung von $[\cdot, \cdot]$ und $\langle\cdot, \cdot\rangle_{\mathbb{B}}$ wird durch $\left(\mathfrak{B}, \mathfrak{Y}, \mathfrak{\mathfrak { B } ^ { \prime }}\right)$ ein Evolutionstripel definiert $[10]$. Auf $\mathfrak{Z}$ werden die Operatoren.

$$
\begin{array}{ll}
\mathscr{A}:=\left(\begin{array}{ll}
0 & -I \\
A & 0
\end{array}\right), \quad & \left(\mathscr{A} \mid \mathfrak{B} \rightarrow \mathfrak{B}^{\prime}\right), \\
\mathscr{A}_{\varepsilon}:=\left(\begin{array}{cc}
0 & -I \\
A & \varepsilon A
\end{array}\right) & \left(\mathscr{A}_{\varepsilon} \mid \mathfrak{B} \rightarrow \mathfrak{B}^{\prime}\right), \\
\mathscr{B}:=\left(\begin{array}{ll}
0 & 0 \\
B_{1} & B_{\mathfrak{a}}
\end{array}\right) & \left(\mathscr{B} \mid \mathfrak{B} \rightarrow\{0\} \times \mathscr{L}_{U}\right)
\end{array}
$$

eingeführt, die für $\varphi \in \mathfrak{B}$ durch

$$
\mathscr{A} \varphi:=\left(\begin{array}{c}
-\varphi_{1} \\
A \varphi_{0}
\end{array}\right), \quad \mathscr{A}_{\iota} \varphi:=\left(\begin{array}{c}
-\varphi_{1} \\
A \varphi_{0}+\varepsilon A \varphi_{1}
\end{array}\right), \quad \mathscr{B} \varphi:=\left(\begin{array}{c}
0 \\
B_{1}(l) \varphi_{0}+B_{2}(t) \varphi_{1}
\end{array}\right)
$$

definiert sind. Mit den Bezeichnungen

$$
\begin{aligned}
& \vec{y}_{\epsilon}(t):=\left(\begin{array}{l}
y_{\varepsilon 0}(t) \\
y_{\varepsilon 1}(t)
\end{array}\right)\left(y_{\varepsilon 0}(t):=y_{\varepsilon}(t), \quad y_{\varepsilon 1}(t):=y_{\epsilon}^{\prime}(t), \vec{y}(t)\right):=\left(\begin{array}{c}
0 \\
g(t)
\end{array}\right) \\
& \text { und } \vec{z}(t):=\left(\begin{array}{c}
0 \\
z(t)
\end{array}\right)
\end{aligned}
$$

kann die Aufgabe (3.1) umgeschrieben werden zu

$$
d \vec{y}_{c}(t)+\mathscr{A}_{c} \vec{y}_{\varepsilon}(t) d t=\vec{g}(t) d \dot{t}+\mathscr{B} \vec{y}_{c}(t) w(d t)+\vec{z}(d t)
$$


mit $\vec{y}_{\varepsilon}(0)=\left(\begin{array}{l}y_{0} \\ y_{1}\end{array}\right)$. Es wird gezeigt, da $\mathbf{B}$ die Gleichung (3.3) die Voraussetzungen zum Satz 1.1 über dem Evolutionstripel ( $\mathfrak{B}, \mathfrak{\mathcal { D }}, \mathfrak{P}^{\prime}$ ) erfiillt.

Wegen [10] gilt

$$
-\langle\mathscr{A} \varphi, \varphi\rangle_{\mathfrak{B}}=\mathbf{0} \text {. }
$$

Somit erhält man $\left\langle\mathscr{A}_{\mathfrak{\varepsilon}} \varphi, \grave{\varphi}\right\rangle_{\mathfrak{B}}=\varepsilon\left\langle A \varphi_{1}, \varphi_{1}\right\rangle_{V}$. Aus dér Voraussetzung $\left(V_{1}\right)$ folgt für beliebige $\varphi \in \mathfrak{B}$ und $\lambda>0$

$$
\begin{aligned}
& 2\left\langle\mathscr{A}_{\varepsilon} \varphi, \varphi\right\rangle_{\mathscr{B}}+\lambda\|\varphi\|_{S^{2}}{ }^{2} \\
& =2 \varepsilon\left\langle A \varphi_{1}, \varphi_{1}\right\rangle_{V}+\lambda\left\langle A \varphi_{0}, \varphi_{0}\right\rangle_{V}+\lambda\left\|\varphi_{1}\right\|_{H}{ }^{2} \\
& \geqq 2 \delta \varepsilon\left\|\varphi_{1}\right\|_{V}^{2}+\lambda \delta\left\|\varphi_{0}\right\|_{V}^{2}+\lambda\left\|\varphi_{1}\right\|_{H}{ }^{2} \geqq \min \{\lambda, 2 \varepsilon\}\|\varphi\|^{2},
\end{aligned}
$$

und daher gilt

$$
2\left\langle-\mathscr{A}_{\iota} \varphi, \varphi\right\rangle_{\mathfrak{B}}+\delta \cdot \min \{\lambda, 2 \varepsilon\}\|\varphi\|_{\mathfrak{B}^{2}} \leqq \lambda\|\varphi\|_{\mathfrak{Q}^{2}} .
$$

Mit der Voraussetzung $\left(V_{2}\right)$ ergibt sich die Koerzitivitätsbedingung $\left(A_{2}\right)$ fiir die Operatoren $\mathscr{A}_{\xi}$ und $\mathscr{B}$ mit $f \equiv 0$. Aus der Voraussetzung $\left(V_{1}\right)$ folgt die Stetigkeit von $\mathscr{A}_{\varepsilon}$, und somit erfüllt $\mathscr{A}_{c}$ insbesondere die Stetigkeitsvoraussetzung $\left(A_{1}\right)$. Aus der Ungleichung (3.5), der Iinearität von $\mathscr{A}_{i}$ und aus $\left(\mathrm{V}_{2}\right)$ folgt für die Operatoren $\mathscr{A}_{\varepsilon}$ und $\mathscr{B}$ die Bedingung $\left(\mathrm{A}_{3}\right) ;\left(\mathrm{A}_{4}\right)$ und $\left(\mathrm{A}_{5}\right)$ sind offensichtlich mit $f \equiv 0$ erfïllt. Die Voraussetzungen $\left(V_{3}\right)$ und $\left(V_{4}\right)$ sichern die Meßbarkeitsbedingungen des' 1 . Kapitels. Die Aussage des Satzes 1.1 liefert nun die Behauptung

Satz 3.2: Es gibt einen bis auf stochastische Modifikationen eindeutig bestimmten. Prozeß $(y(t)) \in \tilde{\mathscr{L}}_{V^{2}}$, der mit Wahrscheinlichkeit 1 der Gleichung (2.1) genügt.

Béwe'is: Aus der Ungleichung (3.2) folgt die Beschränktheit der Familie $\left\{\left(y_{\varepsilon}(t), y_{\varepsilon}^{\prime}(t)\right): \varepsilon>0\right\}$ in $\mathscr{L}_{\mathfrak{Q}^{2}}{ }^{2}$ für $\varepsilon \downarrow 0$. Daher ergibt sich für eine Teilfolge $(\bar{\varepsilon}) \subset(\varepsilon)$ die schwache Konvergenz der Folge $\left(y_{\varepsilon}(t), y_{\varepsilon}^{\prime}(t)\right)$ in $\mathscr{L}_{\mathfrak{s}}{ }^{2}$ gegen ein Element $\left(y(t), y^{\prime}(t)\right) \in \tilde{\mathscr{L}}_{\mathfrak{G}^{2}}$. Durch $\int_{0} B_{i}(s) x(s) w(d s) \quad\left(i=1,2 ; x \in \tilde{\mathscr{L}}_{v^{2}}\right)$ iverden lineare stetige Abbildungen $\Phi$ von $\tilde{\mathscr{L}}_{v^{2}}^{0}$ in $\tilde{\mathscr{L}}_{H^{2}}$, definiert. Aus der Beziehung

$$
\begin{aligned}
& E \int_{0}^{T}\left(\int_{0}^{t} B_{i}(s) w(d s), r(s)\right)_{H} d s \\
& =E \int_{0}^{T} \int_{0}^{t}\left\langle B_{i}(s) x(s) w(d s), r(s)\right\rangle_{V} d s=E \int_{0}^{T}\left\langle E\left\{\Phi_{i}^{*}(r) \mid \widetilde{\mho}_{s}\right\}, x(s)\right\rangle_{V} d s
\end{aligned}
$$

$\left(i=1,2 ; r \dot{\epsilon} \tilde{\mathscr{L}}_{V^{2}}\right.$ beliebig; $\Phi^{*}-\operatorname{der}$ zu $\Phi$ adjungierte Operator) ergibt sich nit $x(s)=y_{\bar{\varepsilon}}(s)$ für $i=1$ und $x(s)=y_{\bar{\varepsilon}}{ }^{\prime}(s)$ für $i=2$ wegen der schwachen Konvergenz von $\left(y_{\bar{\varepsilon}}(t), y_{\bar{\varepsilon}}{ }^{\prime}(t)\right)$ die schwache Konvergenz der Folgen

$$
\left(\int_{0} B_{1}(s) y_{\bar{\varepsilon}}(s) w(d s)\right)_{\bar{\varepsilon}<0}, \backslash \quad\left(\int_{0}^{t} B_{2}(s) y_{\bar{\varepsilon}}{ }^{\prime}(s) w(d s)\right)_{\bar{c}>0}
$$

in $\mathscr{L}_{V}^{2}$. A A us der Beschränktheit der Folge $\left(\gamma_{\bar{\varepsilon}}^{-} y_{\varepsilon}{ }^{\prime}(t)\right)_{\varepsilon>0}$ in $\mathscr{L}_{V}{ }^{2}$ folgt lim $\varepsilon E \int^{T}\left\|A y_{\varepsilon}^{\prime}(t)\right\|_{V}^{2} \cdot d t$ $\doteq 0$. Wegen

$$
E \int_{0}^{T}\left\langle A y_{\bar{\varepsilon}}(s), r(s)\right\rangle_{V} d s=E \int_{0}^{T}\left\langle A^{*} r(s) ; y_{\bar{\varepsilon}}(s)\right\rangle_{V} d s
$$


erhält man die schwache Konvergenz von $A y_{\bar{\varepsilon}}(s)$ gegen $A y(s)$ in $\mathscr{L}_{v}^{2}$. Aus der Gleichung

$$
\begin{aligned}
y_{\bar{\varepsilon}}{ }^{\prime}(t)= & y_{1}-\int_{0}^{t} A y_{\bar{\epsilon}}(s) d s-\varepsilon \int_{0}^{t} A y_{\bar{z}}{ }^{\prime}(s) d s+\int_{0}^{t} g(s) d s \\
& +\int_{0}^{t}\left(B_{1}(s) y_{\bar{c}}(s)+B_{2}(s) y_{\bar{\varepsilon}}(s)\right) w(d s)+z(t)
\end{aligned}
$$

erhält man somit die schwache Konvergenz von $\left(y_{\bar{\varepsilon}}{ }^{\prime}(t)\right)$ in $\mathscr{L}_{V^{*}}^{2}$ für $\bar{\varepsilon} \downarrow 0$ gégen

$$
y^{\prime}(t)=y_{1}-\int_{0}^{t} A y(s) d s+\int_{0}^{t}\left(B_{1}(s) y(s)+B_{2}(s) y^{\prime}(s)\right) w(d s)+z(t)
$$

d. h., $\left(y(l), y^{\prime}(t)\right)$ löst die Gleichung (2.1).

Es bleibt zu zeigen, daß $\left(y(t), y^{\prime}(t)\right)$ mit Wahrscheinlichkeit 1 die einzige Lösung in $\mathscr{L}_{\mathfrak{\xi}}{ }^{2}$ ist. Angenommen $\left(x(t), x^{\prime}(t)\right) \in \mathscr{L}_{\bar{g}^{2}}{ }^{2}$ mit

$$
P\left\{\sup _{i}\left\{\|x(t)-y(t)\|_{V},\left\|x^{\prime}(t)-y^{\prime}(t)\right\|_{H}\right\}>0\right\}>0
$$

löst die Gleichung (2.1). Dann gilt für die Prozesse $\eta(t):=y(t)-x(t), \eta^{\prime}(t)=y^{\prime}(t)$ $-x^{\prime}(t)$ die Beziehung ।

$$
\eta^{\prime}(t)=\int_{0}^{t}(-A \eta(s)) d s+\int_{0}^{t}\left(B_{1}(s) \eta(s)+B_{2}(s) \eta^{\prime}(s)\right) w(d s)
$$

Substituiert man $\vec{\eta}(t):=\left(\begin{array}{l}\eta(t) \\ \eta^{\prime}(t)\end{array}\right)$ und wendet über dem Evolutionstripel $\left(\mathfrak{B}, \mathfrak{j}, \mathfrak{Q}^{\prime}\right)$ die Ttofornicl fiur das Normquadrat [8] auf $\|\eta(t)\|_{\mathfrak{q}^{2}}$ an und beachtet die Formel (3.4) und die Eigenschaft ( $\mathrm{V} 2$ ), so erhält man

$$
\begin{aligned}
E_{\|}\|\vec{\eta}(t)\|_{\mathfrak{Q}^{2}}= & -2 E \int_{0}^{t}\langle\mathscr{A} \vec{\eta}(s), \vec{\eta}(s)\rangle_{\mathfrak{B}} d s \\
& +\int_{0}^{t}\left\|B_{1}(s) \eta(s)+B_{2}(s) \eta^{\prime}(s)\right\|_{Q^{2}} d s \\
\leqq & 2 h E \int_{0}^{t} i\|\vec{\eta}(t)\|_{\mathfrak{S}}{ }^{2} d t .
\end{aligned}
$$

$E\|\vec{\eta}(t)\|_{\Phi^{2}}$ ist bezüglich $t \in[0, T]$ eine beschränkte Funktion. Mit dem Gronwallschen Temria folgt aus der letzten Ungleichung $E\|\vec{\eta}(t)\|_{\Phi^{2}} \leqq 0$ für alle $t \in[0, T]$, d. h. $E\left\{\langle A \eta(t), \eta(t)\rangle_{V}+\left\|\eta^{\prime}(t)\right\|_{H^{2}}\right\} \leqq 0$. Hieraus folgt mit der Voraussetzung $\left(\mathrm{V}_{1}\right) \eta(t)=0$, $\eta^{\prime}(t)=0$ (fast sicher), d. h. $\left(y(t), y^{\prime}(t)\right)$ ist bis auf stochastische Modifikationen eindeutig bestimmt

Satz 3.3: Esigilt

$$
\lim _{\varepsilon \downarrow 0}\left[E \sup _{t}\left(\left\|y_{e}(t)-y(t)\right\|_{v^{2}}+\left\|y_{e}^{\prime}(t)-y^{\prime}(t)\right\|_{H^{2}}\right)\right]=0 .
$$


Beweis: Wegen den Sätzen 3.1 und 3.2 existieren mit Wahrscheinlichkeit 1 eindeitig bestimmte Lösungen $\left(y_{\varepsilon}(t)\right),(y(t)) \in \tilde{\mathscr{L}}_{V^{2}}$ der Gleichungen (3.1) und (2.1). Fïr

$$
\vec{\eta}_{\varepsilon}(t):=\left(\begin{array}{c}
\eta_{\varepsilon}(t) \\
\eta_{\varepsilon}^{\prime}(t)
\end{array}\right):=\left(\begin{array}{c}
y_{\varepsilon}(t)-y(t) \\
y_{\varepsilon}^{\prime}(t)-y^{\prime}(t)
\end{array}\right)
$$

gilt in der Bezeichnungsweise zum Beweis des Säzes 3.1 die Glèichung

$$
d \vec{\eta}_{\varepsilon}(t)+\mathscr{A}_{\varepsilon} \vec{\eta}_{\varepsilon}(t) d t+\varepsilon\left(\begin{array}{c}
0 \\
A y(t)
\end{array}\right) d t=\mathscr{B} \vec{\eta}_{\varepsilon}(t) w(d t)
$$

mit $\vec{\eta}_{\varepsilon}(0)=\left(\begin{array}{l}0 \\ 0\end{array}\right)$. Fiir $\varphi \in \mathfrak{B}$ gilt

$$
\varepsilon\|A y(t)\|_{V^{\bullet}}\|\varphi\|_{\mathfrak{B}} \leqq \frac{1}{\delta} \frac{1}{2} \varepsilon\|A y(t)\|_{V}^{2}+\delta \frac{1}{2} \varepsilon\|\varphi\|_{\mathfrak{B}^{2}}
$$

Somit erhält miân

$$
\begin{aligned}
\varepsilon\left\langle(0, A y(t)),\left(\begin{array}{c}
\dot{\varphi}_{0} \\
\varphi_{1}
\end{array}\right)\right)_{\mathfrak{B}} & \geqq-\varepsilon\left|\left\langle(0, A y(t)),\left(\begin{array}{c}
\varphi_{0} \\
\varphi_{1}
\end{array}\right)\right\rangle_{\mathfrak{B}}\right| \geqq-\varepsilon\left|\left\langle A y(t) ; \varphi_{1}\right\rangle_{V}\right| \\
& \geqq-\varepsilon\|A y(t)\|_{V^{*}}\left\|\varphi_{1}\right\|_{V} \geqq-\varepsilon\|A y(t)\|_{V^{*}}\|\varphi\|_{\mathfrak{B}} \\
& \geqq-\frac{\varepsilon}{2 \delta}\|A y(t)\|_{V^{*}}^{2}-\frac{\varepsilon \cdot \delta}{2}\|\varphi\|_{\mathfrak{B}^{2}} .
\end{aligned}
$$

Mit der Ungleichung (3.5) ergibt sich somit

$$
\begin{aligned}
& 2\left\langle\mathscr{A}_{\varepsilon} \varphi, \varphi\right\rangle_{\mathfrak{B}}+2 \varepsilon\left\langle(0, A y(t)),\left(\begin{array}{c}
\varphi_{0} \\
\varphi_{1}
\end{array}\right)\right\rangle_{\mathfrak{B}}-\lambda\|\varphi\|_{\mathfrak{S}^{2}} \\
& \geqq \delta \min \{\lambda, \dot{2} \varepsilon\}\|\varphi\|_{\mathfrak{B}^{2}}-\frac{\varepsilon}{\delta}\|A y(t)\|_{V^{*}}^{2}, \varepsilon \delta\|\varphi\|_{\mathfrak{B}^{2}} \\
& =(\delta \cdot \min \{\lambda, 2 \varepsilon\}-\varepsilon \delta)\|\varphi\|_{\mathfrak{B}^{2}} .
\end{aligned}
$$

Hieraus folgt

$$
\begin{aligned}
& 2\left\langle-\dot{A}_{,} \varphi, p\right\rangle_{\mathfrak{B}}+2\left\langle-(0, \cdot \varepsilon A y(t)),\left(\begin{array}{c}
\dot{\varphi}_{0} \\
\varphi_{1}
\end{array}\right)\right\rangle_{\mathfrak{B}}+(\delta \min \{\dot{\lambda}, \dot{2} \dot{\varepsilon}\}-\delta \varepsilon)\|\varphi\|_{\mathfrak{B}^{2}} \\
& \leqq \lambda\|\varphi\|_{\mathfrak{Q}^{2}}+\frac{\varepsilon}{\delta}\|A y(t)\|_{V^{*}}^{2}
\end{aligned}
$$

Fïr hinreichend klcines $\varepsilon$ ist $\delta \min \{\dot{\lambda}, 2 \varepsilon\}=2 \varepsilon \delta$. Damit ist der Faktor vor $\|\varphi\|_{\mathfrak{B}^{2}}$ positiv. Unter Beachtung der Voraussetzungen $\left(V_{2}\right)-\left(V_{4}\right)$ ergibt sich für hinreichend kleine $\varepsilon$ das Erfülltsein der Voraussetzungen zum Satz $1.1 \mathrm{mit} f(t)=\frac{\dot{\varepsilon}}{\delta}\|\dot{A} y(t)\|_{V^{*}}^{2}$,

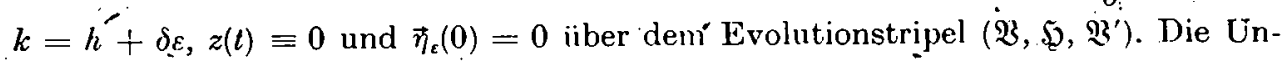
gleichung (1.2) zeigt

$$
E \sup _{0 \leqq i \leqq T}\left[\left\|y_{\varepsilon}(t)-y(t)\right\|_{V^{2}}{ }^{-}+\left\|y_{c}^{\prime}(t)-y^{\prime}(t)\right\|_{h^{2}}\right] \leqq \frac{\varepsilon}{\delta} E \int_{0}^{T}\|A y(t)\|_{v^{*}}^{2} d t .
$$

Fiir $\dot{\varepsilon} \downarrow 0$ ergibt sich die Behauptung 
Folgerung: Der Proze $\beta\left(y(t), y^{\prime}(t)\right)$ ist als $V \times H$-werliger Proze $\beta$ mit Wahrscheinlichkeit 1 stetig.

Beweis: Aus dem Satz 3.3 folgt

$$
\lim _{\delta \downarrow 0} P\left\{\sup _{0 \leqq t \leqq T}\left[\left\|y_{\varepsilon}(t)-y(t)\right\|_{\nabla^{2}}+\left\|y_{\varepsilon}^{\prime}(t)-\hat{y}^{\prime}(t)\right\|_{h^{2}}\right]>\gamma\right\}=0
$$

fiir alle $\gamma>0$. Da aus der Konvergenz in Wahrscheinlichkeit die Konvergenz mit Wahrscheinlichkeit 1 für eine Teilfolge folgt, gibt es für fast alle $\omega \in \Omega$ Folgen $\varepsilon_{n}:=\varepsilon_{n}(\omega) \subset(\varepsilon)$, so daß

$$
P\left\{\lim _{\varepsilon_{n} \downarrow 0} \sup _{0 \leqq t \leq T}\left[\left\|y_{\varepsilon_{n}}(t)-y(t)\right\|_{V^{2}}+\left\|y_{\varepsilon_{n}}^{\prime}(t)-y^{\prime}(t)\right\|_{H^{2}}{ }^{2}=0\right\}=1,\right.
$$

gilt, d. h. $\left(y_{\varepsilon_{n}}(t), y_{\varepsilon_{n}}^{\prime}(t)\right)$ konvergiert für fast alle $\omega \in \Omega$ gleichn̈̈ßig bezüglich $t \in[0, T]$ gegen $\left(y(t), y^{\prime}(t)\right)$ im Raum $V \times H$. Da aus dem Satz 1.1 die Stetigkeit von $\left(y_{\varepsilon_{n}}(t)\right.$, $\left.y_{\varepsilon_{n}}^{\prime}(t)\right)$ bezüglich $t \in[0, T]$ auf $\Omega$ als $V \times H$-wertige Funktion folgt, ist somit $\left(y(t), y^{\prime}(t)\right)$ realisierungsweise stetig in $V \times H$

Bemerkung 3.5: Die Sätze 3.1 bis 3.3 gelten insbesondere für $B_{1} \equiv 0, B_{2} \equiv 0$ und $z \equiv 0$. Man erhält dann den in [10] betrachteten deterministischen Fall.

Bemerkung 3.6: Die Aussagen der Sätze 3.1 bis 3.3 gelten auch - mit Ausnahme der $\widetilde{F}_{t}$-Meßbarkeit - für $B_{1} \equiv 0, B_{2} \equiv 0$ und $(z(t)) \in \tilde{\mathscr{L}}_{H}{ }^{2}$, wenn die Anfangsbedingungen, durch Endbedingungen ersetzt werden.

\section{Eine Anwendung in der Steuertheorie}

Es seien $D$ eine konvexe abgeschlossene Menge in $H$ und

$$
\mathscr{D}:=\left\{(u(t)): u \mid[0, T] \times \Omega \rightarrow D,\left(\mathfrak{F}_{t}, \mathfrak{B}_{H}\right) \text {-meBbar, } E \int_{0}^{T}\|u(t)\|_{H}{ }^{2} d t<\infty\right\},
$$

$W$ ein separabler Hilbertraum, $C(t) \in L(H, W), N \in L(H, H)$ mit $(N x, x) \geqq v\|x\|_{H}{ }^{2}$ fiir alle $x \in H, x_{0}$ ein Element aus $W$. Fiir $K=H$ wird die Steueraufgabe

$$
\left\{\begin{array}{l}
\dot{d y^{\prime}}(t)+A y(t) d t=u(t)+u:(d t), y(0)=y_{0} \in V, y^{\prime}(0)=y_{1} \in H \\
J(u):=F \int_{0}^{T}\left\|C(t) y(t)-x_{0}\right\|_{w^{2}} d t+E \int_{0}^{T}(N u(t), u(t))_{H}=\operatorname{Min}_{u \in \mathscr{D}}
\end{array}\right.
$$

betrachtet. Ihr wird mit der Bezeichnungsweise des 2. Kapitels die Aufgabe

$$
\left\{\begin{array}{l}
d \vec{y}_{\epsilon}(t)+\dot{\mathscr{A}}_{\varepsilon} \vec{y}_{\varepsilon}(t) d t=\vec{u}(t) d t+\vec{w}(d t), \vec{y}_{\varepsilon}(0)=\left(\begin{array}{l}
y_{0} \\
y_{1}
\end{array}\right) \\
J_{\varepsilon}(u)=E \int_{0}^{T}\left\|\mathscr{C} \vec{y} y_{e}(t)-x_{0}\right\|_{w} d t+E \int_{0}^{T}(N u(t), u(t))_{H} d t \dot{\prime}^{\prime} \operatorname{Min}_{u \in \mathscr{Q}}
\end{array}\right.
$$

zugeordnet, wobei für $\varphi \in \mathfrak{g}$ die Abbildung $\mathscr{C} \mid \mathfrak{Q} \rightarrow W$ durch $\mathscr{C} \varphi=C^{\prime}(\ell) \varphi_{0}$ definiert . ist.

Aus den Voraussetzungen folgt, da $\beta$ fïr die Steuéraufgaben (4.1), (4.2) und $\left(4.1_{\iota}\right)$, $\left(4.2_{\varepsilon}\right)$ optimale Steuerungen $\left(u^{0}(t)\right),\left(u^{0}(t)\right) \in \mathscr{D}$ existieren. $\left(y^{0}(t)\right)$ und $\left(y_{\varepsilon}{ }^{0}(t)\right)$ bezeichnen die zu den Steuerungen $\left(u^{0}(t)\right)$ und $\left(u_{\varepsilon}^{0}(t)\right)$ gehörigen Tïösungen der Gleichungen (4.1) und $\left(4.1_{\varepsilon}\right)$. Die Gleichung $\left(4.1_{\varepsilon}\right)$ ist wegen des Satzes 3.1 eine parabolische 


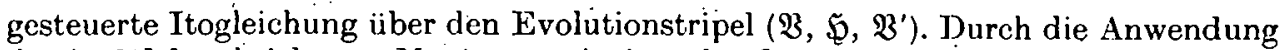
des in [1] beschriebenen Maximumprinzipes für Steueraufgaben mit parabolischen Itogleichungen erhält man, da $\beta\left(u_{\varepsilon}^{0}(t)\right) \in \mathscr{D}$ optimal für $\left(4.1_{\varepsilon}\right),\left(4.2_{\varepsilon}\right)$ genau dann ist, wenn für alle $(u(t)) \in \mathscr{D}$ mit Wahrscheinlichkeit 1 gilt

$$
\left(-E\left\{p_{\varepsilon 1}^{0}(t) \mid \mathfrak{F}_{t}\right\}+2 N u_{\varepsilon}^{0}(t), u(t)-u_{\varepsilon}^{0}(t)\right)_{H} \geqq 0,
$$

wobei $\vec{p}_{\varepsilon}^{0}(t):=\left(\begin{array}{l}p_{\varepsilon 0}^{0}(t) \\ p_{\varepsilon 1}^{1}(t)\end{array}\right)$ realisierungsweise die Gleichung

$$
-\frac{d \vec{p}_{\varepsilon}{ }^{0}(t)}{d t}+\mathscr{A}_{\varepsilon}^{*} \vec{p}_{\varepsilon}{ }^{0}(t)=2 \mathscr{C} *\left(\mathscr{C}_{\vec{y}}(t)-x_{0}\right)
$$

mit $\vec{p}_{\varepsilon}^{0}(T)=\left(\begin{array}{l}0 \\ 0\end{array}\right)$ löst (*1 - Zeichen für den adjungierten Operator $)$. Für beliebige $\varphi \in \mathfrak{W}, \psi \cdot \in \cdot W$ gilt

$$
\left(\mathscr{C}^{*} \psi, \varphi\right)_{\mathfrak{Q}}=(\mathscr{C} \varphi, \psi)_{W}=\left(C(t) \varphi_{0}, \psi\right)_{W,}=\left\langle C^{*}(t) \psi, \varphi_{0}\right)_{H}=\left\langle C^{*}(t) \psi, \varphi_{0}\right\rangle_{V}
$$

Aus der Definition des Skalarproduktes iiber $\mathfrak{W}$, folgt, daß der letzte Ausdruck in der Gestalt $\langle A \cdot, \cdot\rangle_{V}$ darstellbar sein muß, d. 市., $\dot{\mathscr{C}}^{*} \psi=\left(\begin{array}{c}A^{-1} C(\ell) \psi \\ 0\end{array}\right)$. Somit erhält man
für die Gleichung (4.4)

$$
-\frac{d p_{\varepsilon}{ }^{0}(t)}{d t}+\mathscr{A}_{\varepsilon}^{*} p_{\varepsilon}{ }^{0}(t)=\left(\begin{array}{c}
2 A^{-1} C^{*}(t)\left[C y_{\varepsilon}(t)-x_{0}\right] \\
0
\end{array}\right) .
$$

Unter Beachtung der Beziehung $\mathscr{A}^{*}=-\mathscr{A}$ erhält man fïr die Gleichung (4.4)

$$
\begin{array}{ll}
-p_{\varepsilon 0}^{0^{\prime}}(t)+p_{\ell 1}^{0^{\prime}}(t)=2 A^{-1} C^{*}(t)\left[C y_{\varepsilon}(t)-x_{0}\right], & p_{\varepsilon 0}^{0}(T)=0, \\
-p_{\varepsilon 1}^{0^{\prime}}(t)-A p_{\varepsilon 0}^{0}(t)+\varepsilon \cdot A p_{\varepsilon 1}^{0}(t)=0, \quad & p_{\varepsilon 1}^{0}(T)=0,
\end{array}
$$

wobei die Gleichungen mit Wahrscheinlichkeit 1 für alle $t \in\left[0, T^{\prime}\right]$ gelten. Aus der Gleichung (4.6). folgt $p_{\varepsilon 1}^{0^{\prime \prime}}(t)-A p_{\varepsilon 0}^{0^{\prime}}(t)+\varepsilon A p_{c 1}^{0^{\prime}}(t)=0$. Mit der Gleichung (4.5) folgt hieraus für $p_{\varepsilon}^{0}(t):=p_{\varepsilon 1}^{0}(t)$ die realisierungsweise Beziehung

$$
p_{\varepsilon}{ }^{0^{\prime \prime}}(t)+A p_{\varepsilon}{ }^{0}(t)-\varepsilon A p_{\varepsilon}{ }^{0^{\prime}}(t)=2 C^{*}(t)\left[C(t) y_{\varepsilon}(t)-x_{0}\right]
$$

mit $p_{\varepsilon}{ }^{0}(T)=10, p_{\varepsilon}{ }^{0}(T) \doteq 0$.

\section{Satz 4.1: Es gelten die folgenden Grenzwertbeziehungen:}

a) $\lim _{t \downarrow 0} E \int_{0}^{T}\left\|u_{c}^{0}(t)-u^{0}(t)\right\|_{H}^{2} d t=0$

b) $\lim _{\epsilon \downarrow 0} E \int_{0}^{T}\left[\left\|y_{\varepsilon}{ }^{0}(t)-y^{0}(t)\right\|_{v^{2}}+\left\|y_{\varepsilon}{ }^{0^{\prime}}(t)-y^{0^{\prime}}(t)\right\|_{H^{2}}{ }^{2}\right] d t=0$;

c) $\lim _{\varepsilon \downarrow 0} J_{\varepsilon}\left(u_{t}^{0}\right)=J\left(u^{0}\right)$.

Beweis: Wegen der Stetigkeitsvoraussetzungen an $J$ und des Satzes 3.3 gilt für alle $(u(t)) \in \mathscr{D} \lim _{\varepsilon \downarrow 0} J_{\varepsilon}(u)=J(u)$. Insbesondere gilt dann $J_{\varepsilon}\left(u_{\varepsilon}^{0}\right) \leqq J_{\epsilon}\left(u^{0}\right) \underset{\varepsilon \downarrow 0}{\longrightarrow} J\left(u^{0}\right)$ und somit

$$
\varlimsup_{\varepsilon \downarrow 0} J_{\varepsilon}\left(u_{\varepsilon}^{0}\right) \leqq J\left(u^{0}\right) .
$$

Nach Voraussetzung gilt $J_{\varepsilon}\left(u_{\varepsilon}^{0}\right) \geqq v E \int_{0}^{T}\left\|u_{\varepsilon}^{0}(t)\right\|_{H}^{2} d t$. 
Aus der,Beziehung (4.8) folgt damit die Beschränktheit von $\left\{\left(u_{\varepsilon}^{0}(t)\right): \varepsilon>0\right\}$ im Raum $\tilde{\mathscr{L}}_{H^{2}}{ }_{\tilde{\tilde{L}}}$ Daher gibt es $(\bar{\varepsilon}) \subset(\varepsilon)$, so da $B\left(u_{\bar{\varepsilon}}{ }^{0}(t)\right)$ schwach in $\mathscr{L}_{H}{ }^{2}$ gegen einen Proze $B$ $(\xi(t))$ aus $\tilde{\mathscr{L}}_{H^{2}}{ }^{2}$ konvergiert. Aus dem Beweis zum Satz 3.2 folgt die schwache Konvergenz von $\left(y_{\bar{c}}^{0}(t)\right)$ gegen $\left(y^{\xi}(t)\right)$ und von $\left(y_{\bar{z}}^{\mathbf{0}^{\prime}}(t)\right)$ gegen $\left(y^{\xi^{\prime}}(t)\right)$ in $\mathscr{L}_{V^{2}}$ und $\mathscr{L}_{H}{ }^{2}$, .wobei $\left(y^{\xi}(t)\right)$ Lösung der Gleichung (4.1) für $u(t)=\xi(t)$ ist. Weil aus der Konvexität und der Stetigkeit von $J$ die schwache Halbstetigkeit nach unten folgt, erhält man $\varliminf_{\bar{i} \downarrow 0} J_{\bar{\imath}}\left(u^{0}\right) \geqq J(\xi)$. Mit (4.8) folgt hieraus $\lim _{\varepsilon \downarrow 0} J_{\varepsilon}\left(u_{\varepsilon}^{0}\right)=J\left(u^{0}\right)$.

In der Beziehung

$$
\left|J\left(u^{0}\right)-J\left(u_{\varepsilon}^{0}\right)\right| \leqq\left|J\left(u^{0}\right)-J_{\varepsilon}\left(u_{\varepsilon}^{0}\right)\right|+\left|J_{\varepsilon}\left(u_{\varepsilon_{0}^{0}}^{0}\right)-J\left(u_{\varepsilon}^{0}\right)\right|
$$

konvergiert der erste Summand wegen c) gegen Null. Wegen des Satzes 3.3 und der Stetigkeit von $J$ fällt der zweite Summand beliebig klein aus. Somit ist $\left(u_{\varepsilon}{ }^{0}\right)$ eine Minimalfolge. Aus den Voraussetzungen ergibt sich sonıit die starke Konvergenz von $\left(u_{\varepsilon}^{0}(t)\right)$ in $\mathscr{L}_{H}^{2}$ gegen $\left(u^{0}(t)\right)$, d. h. es gilt a). Aus dem Satz 3.3 folgt dann b)

Bemerkung: Der letzte Satz zeigt, daß die optimalen Steuerungen der parabolisch regularisierten Aufgabe $\left(4.1_{\varepsilon}\right) ;\left(4.2_{\varepsilon}\right)$ Näherungen für die Aufgabe (4.1), (4.2) liefern.

Aus der Aussage b) in Satz 4.1 und der Bemerkung 3.6 folgt

Satz 4.2: Es gilt

$$
\lim _{\varepsilon \downarrow 0} E \int_{0}^{T}\left(\left\|p_{\varepsilon}{ }^{0}(t)-p^{0}(t)\right\|_{V^{2}}+\left\|p_{\varepsilon}^{0^{\prime}}(t)-p^{0^{\prime}}(t)\right\|_{H}{ }^{2}\right) d t=0
$$

wobei $\left(p^{0}(t)\right)$ Lösung im Sinne der Bemerkung 3.6 der Aufgabe

$$
p^{0^{\prime \prime}}(t)+A p^{0}(l)=2 C^{*}(t)\left[C(t) y^{0}(t)-x_{0}\right]
$$

mit $p^{0}\left(T^{\prime}\right)=0, p^{0^{\prime}}\left(T^{\prime}\right)=0$ ist.

Man erhält also

Satz 4.3: $\left(u^{0}(t)\right) \in \mathscr{D}$ ist genau dann optimale Steuerung für-die Aufgabe:(4.1), (4.2), wenn ein $\left(p^{0}(t)\right) \in \mathscr{L}_{V^{2}}$. existiert, so daß die Gleichung (4.9) realisierungsweise gilt und für alle $(u(t)) \in \mathscr{D}$

$$
\text { - }\left(-E\left\{p^{0}(t) \mid \Im_{t}\right\}+2 N u^{0}(t), u(t)-u^{0}(t)\right)_{H} \geqq 0
$$

mit Wahrscheinlichkeit 1 . und alle $t \in[0, T]$ gilt.

Bemerkung 4.4: Im. Falle $D=H$ ist $u_{\varepsilon}{ }^{0}(t)=\frac{1}{2} N^{-1} E\left\{p_{\varepsilon}^{0}(t) \mid \Im_{t}\right\}$ und $u_{0}^{0}(t)$ $=\frac{1}{.2} N^{-1} E_{i}\left\{p^{0}(t) \mid \xi_{\ell}\right\}$.

Bemerkung 4.5: Die Auswertung des Maximumprinzipes im Satz 4.3 ist wegen des Auftretens der bedingten Érwartung im Ausdruck (4:8) und.der zufälligen rechten Seite in der Gleichung (4.7) șchwièrig. Im Fall „,kleiner Störungen $\gamma w(d t)$ " ( $\gamma$-kleiner positiver Parameter) erhält man

Satz 4.6: Die optimale. Steuerung $\left(u_{00}^{0}(t)\right)$ des deterministischen Problems

$$
\begin{aligned}
& d y^{\prime}(t)+A y(t) d t=u(t) d t, y(0)=y_{0}, y^{\prime}(0)=y_{1},
\end{aligned}
$$

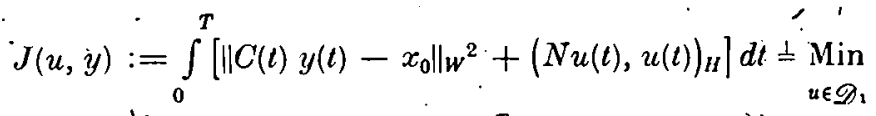

$$
\begin{aligned}
& \left(\dot{D}_{1}:=\left\{(u(t)): u \mid[0, T] \rightarrow D, \int_{0}^{T}\|u(t)\|_{H^{2}} d t<\infty\right\}\right)
\end{aligned}
$$


ist $\gamma$-optimal für die Aufgabe

$$
\begin{aligned}
& d y^{\prime}(t)+A y(t) d t=u(t) d t+\gamma w(d t), y(0)=\dot{y}_{0}, y^{\prime}(0)=y_{1}^{\prime},- \\
& J(u, y)=E \int_{0}^{T}\left[\left\|C(t) y(t)-x_{0}\right\|_{W^{2}}+(N u(t), u(t))_{H}\right] d t \stackrel{!}{=} \underset{u \in \mathscr{D}}{\operatorname{Min}}
\end{aligned}
$$

d. h. es gibt ein $\gamma_{0}>0$, so daß für $\left.\left.\dot{\gamma} \in\right] 0, \gamma_{0}\right]$ gill

$$
0 \leqq J\left(u_{00}^{0}, y_{\gamma 0}\left(u_{00}^{0}\right)\right)-J\left(u_{\gamma 0}^{0}, y_{y 0}\left(u_{y 0}^{0}\right)\right) \leqq c \gamma .
$$

Dabei ist $\left(y_{y 0}\left(u_{00}^{0}\right)\right)$ Lösung der Gleichung (4.13)für $u=u_{00}^{0},\left(u_{y 0}^{0}(t)\right) \in \mathscr{D}$. optimule Steuerung für (4.13)-(4.14), $\left(y_{y 0}\left(u_{y 0}^{0}\right)\right)$ Lösung der Geichung (4.13) für $u=u_{y 0}^{0}$ und $c$ eine von $\gamma$ unabhängige Konstante:

Beweis: Es wird betrachtet

$$
\begin{aligned}
& d \vec{y}_{\varepsilon}(t)+\mathscr{A}_{c} \vec{y}_{\varepsilon}(t) d t=\vec{u}(t) d t+\gamma \dot{w}(d t), \vec{y}_{\varepsilon}(0)=\left(\begin{array}{l}
y_{0} \\
y_{1}
\end{array}\right) \\
& J\left(u, \vec{y}_{\varepsilon}\right)=E \int_{0}^{T}\left\|\mathscr{C}(t) \vec{y}_{\varepsilon}(t)-\vec{x}_{0}\right\|_{w}{ }^{2} d t+E \int_{0}^{T}(N u(t), u(t))_{H} d t \stackrel{\dot{=}}{\operatorname{Min}}
\end{aligned}
$$

Es bezeichnen $\left(u_{y \varepsilon}^{0}(t)\right)$ die optimale Steuerung von $(4.15)-(4.16), \dot{y}_{y \varepsilon}\left(u_{y \varepsilon}^{0}\right)$ die Lösung von (4.15) fïr $u=u_{\gamma e}^{0},\left(u_{0 e}^{0}(t)\right)$ die optimale (deterministische) Steuerung von (4.15) bis (4.16). für $\gamma^{\prime}=0, y_{0 \varepsilon}\left(u_{0 \varepsilon}^{0}\right)$ die Lösung von (4.15) für $u=u_{0 \varepsilon}^{0}$. Offensichtlich gilt

$$
\begin{aligned}
& \left|J\left(u_{\gamma 0}^{0}, y_{y 0}\left(u_{y 0}^{0}\right)\right)-J\left(u_{00}^{0}, y_{\gamma 0}\left(u_{00}^{0}\right)\right)\right| \\
& \leqq\left|J\left(u_{y 0}^{0}, y_{\gamma 0}\left(u_{y 0}^{0}\right)\right)-J\left(u_{y \varepsilon}^{0}, y_{y \varepsilon}\left(u_{y \varepsilon}^{0}\right)\right)\right| \\
& ++\left|J\left(u_{\gamma \varepsilon}^{0}, y_{\gamma \varepsilon}\left(u_{\gamma \varepsilon}^{0}\right)\right)-J\left(u_{0 \varepsilon}^{0}, y_{0 \varepsilon}\left(u_{0 \varepsilon}^{0}\right)\right)\right| \\
& +\left|J\left(u_{0 \varepsilon}^{0}, y_{0 \varepsilon}\left(u_{0 \varepsilon}^{0}\right)\right)-J\left(u_{00}^{0}, y_{00}\left(u_{00}^{0}\right)\right)\right| \\
& +\left|J\left(u_{00}^{0}, y_{00}\left(u_{00}^{0}\right)\right)-J\left(u_{00}^{0}, y_{\gamma 0}\left(u_{00}^{0}\right)\right)\right| \text {. }
\end{aligned}
$$

Für hinreichend kleine $\varepsilon$ fällt wegen des Satzes 4.1 der Ausdruck (4.17) kleiner als $j$ aus. Wegen [7] ist $\left(u_{0 \ell}^{0}(t)\right)$ eine $\gamma$-optimale Steuerung von $(4.15)-(4.16), \mathrm{d}$. h. der Summand (4.18) fällt für hinreichend kleine $\gamma$ kleinér als const $\gamma$ aus, wobei const von $\gamma$ unabhängig ist. Aus der deterministischen Theorie [15] folgt für $\varepsilon \downarrow 0$ die Konvergenz von (1.19) gegen Null. Fïr $\vec{Z}_{y}(t):=\left(\begin{array}{l}y_{\gamma 0}(t)-y_{00}(t) \\ y_{\gamma 0}^{\prime}(t)-y_{00}^{\prime}(t)\end{array}\right)$ gilt $d \vec{Z}_{\gamma}(t)$ $+\mathcal{A} \vec{Z}_{y}(t) d t=\gamma w(d t)$. Mit der Itoformel erhält man unter Beriicksichtigung der Fprmel (3.4)

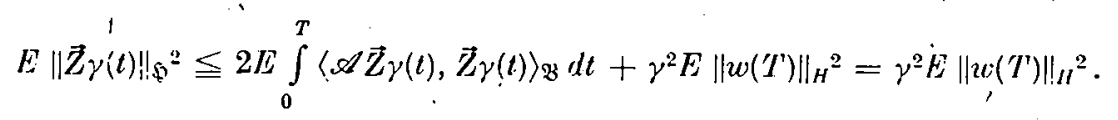

Folglich kònvergiert der Term (4.20) für $\gamma \downarrow 0$ gegen Null. Somit gilt für hinreichend kleine' $\varepsilon$ und $y$ die Uingleichung

$$
\left|J\left(u_{y 0}^{0}, y_{\gamma 0}\left(u_{y_{0} 0}^{0}\right)\right)-J\left(u_{00}^{0}, y_{y 0}\left(u_{00}^{0}\right)\right)\right| \leqq c \gamma,
$$

wobei $c$ eine von $\gamma$ unabhängige Konstante ist 
Beispiel 4. 7: Es sei $D=L^{2}(G)$ und $\Delta$ der Laplaceoperator. Ưber dem Evolutionstripel $\left.\left(\dot{W}_{2}^{1} G\right), L^{2}(G), W_{2}^{-1}(G)\right) \mathrm{mit} G=[0,1] \times[0,1]$ ist die Steueraufgabe

$$
\begin{gathered}
d\left[\frac{\partial y\left(t ; x_{1}, x_{2}\right)}{\partial t}\right]-\Delta y\left(t ; x_{1}, x_{2}\right) d t=u(t) d t+y w(d t), \\
y\left(0 ; x_{1}, x_{2}\right)=y_{0}\left(x_{1}, x_{2}\right),\left.\frac{\partial y\left(t ; x_{1}, x_{2}\right)}{\partial t}\right|_{t=0} \\
=y_{1}\left(x_{1}, x_{2}\right),\left.y\left(t ; x_{1}, x_{2}\right)\right|_{\left(x_{1}, x_{2}\right) \in \partial G}=0
\end{gathered}
$$

(vgl. Bemertung 2.6),

$$
\begin{aligned}
J(u, y)= & E \int_{0}^{T} \int_{G}^{-}\left|y\left(t ; x_{1}, x_{2}\right)-x_{0}\left(x_{1}, x_{2}\right)\right|^{2} d x_{1} d x_{2} d t \\
& +E \int_{0}^{T} \int_{G}\left|u\left(t ; x_{1}, x_{2}\right)\right|^{2} d x_{1} d x_{2} d t \stackrel{\dot{ }}{=} \cdot \operatorname{Min}
\end{aligned}
$$

ein Beispiel für das'System (4.13), (4.14). Die Lösung der deterministischen Aufgabe

$$
\frac{\left.\partial^{2} y t ; x_{1}, x_{2}\right)}{\partial t^{2}}-\Delta y\left(t ; x_{1}, x_{2}\right)=u\left(t ; x_{1}, x_{2}\right)
$$

$\left(y\left(t ; x_{1}, x_{2}^{\prime}\right)\right.$ erfüllt die Randbedingungen (4.22)),

$$
\begin{aligned}
J(u, y)= & \int_{0}^{T} \int_{G}\left|y\left(t ; x_{1}, x_{2}\right)-\grave{x_{0}}\left(x_{1}, x_{2}\right)\right|^{2} d x_{1} d x_{2} d t \\
& +\int_{0}^{T} \int_{G}\left|u\left(\iota ; x_{1}, x_{2}\right)\right|^{2} d x_{1} d x_{2} d t=\underset{u \in \mathscr{Q}_{1}}{\operatorname{Min}}
\end{aligned}
$$

ist' $y$-optimal für (4.21)-(4.23). Für die optimale Steuerúng des deterministischen Systems erhält man

$$
u_{00}^{0}\left(t ; x_{1}, x_{2}\right)=\frac{1}{2} p^{0}\left(t ; x_{1}, x_{2}\right),
$$

wobei gilt

$$
\begin{aligned}
& \frac{\partial^{2} p^{0}\left(t ; x_{1}, x_{2}\right)}{\partial t^{2}}-\Delta p^{0}\left(\ell ; x_{1}, x_{2}\right)=2\left(y_{00}^{0}\left(t ; x_{1}, x_{2}\right)-x_{0}\left(x_{1}, x_{2}\right)\right) \\
& p^{0}\left(T ; x_{1}, x_{2}\right)=0,\left.\frac{\partial p^{0}\left(t ; x_{1}, x_{2}\right)}{\partial t}\right|_{t=T}=0,\left.\quad p^{0}\left(t ; x_{1}, x_{2}\right)\right|_{\left(x_{1}, x_{2}\right) \in \hat{\partial} G=0 .}=0 .
\end{aligned}
$$

\section{I.ITERA'TUR}

[1] Becher, H., und W. Greckscir: Anwendung der Dualitätstheorie in der stochastischen Steuertheorie. Math. Nachr. 115 (1983).

[2] Bexsoussan, A.: Control of stochastic differential equations. In: Control and Systems Theory 6 (Ed.: W. H. Ray), Dekker Publishers: New York 1978, 210-247.

[3] Chajowowsa-Michalik, A.: Stochastic differential equations in Hilbert spaces. In: Banach Center Publications 5, PWN - Polish Scientific Publishers: Warszawal 1979, $53-74$. 
[4] Curtais, R., and A. J. Pritchard: Infinite dimensional linear systems theory (Lecture notes in control and informations sciences Vol. 8). Springer.Verlag: Berlin - HeidelbergNew York 1978.

[5] Гихман, И. И.: Решение иачально-нраевой задачи для стохастического уравнения гиперболического типа методом фурье. Теория случ. проц. 8 (1980), 31-35.

[6] Гихмлн, И. И.: О-первой начально-краевой залаче для стохастического гипероолнческого уравнения. Теория случ. проц. $8(\mathbf{1 9 8 0}) ; 20-31$.

[7] Grecksch. W.: Eine Steueraufgabe für eine parabolische Itogleichung. Tagungsmaterial des 27. Intern. Wiss. Koll. TH Ilmenau 1982, Vortragsreihe ,Mathematische Optimierung - Theorie'und Anwendung“", 87-90.

[8] Крылов, Н. В., и В. И. Розовский: О случайных эволюционных уравнениях. В сб.: Итоги науки и техники 14. Изд-во ВИНИ'ТИ: Москва 1979, 71-146.

[9] ЛАдыженскля, О. А.: Краевые задачи математической физики. Изд-во Наука: Москва 1973.

[10] Lioss, J. L.: Optimal control of systems gouverned by partial differential equations. Springer: Verlag: Berlin-Heidelberg-New York 1971.

[11] Lions, J. L., and E. Magenes: Non homogeneous boundary value problems and applications I. Springer-Verlag: Berlin-Heidelberg-New York 1972.

[12] Metivier, M., and G. Pistone: Une formule d'isometrie pour l'integrale stochastique hilbertienne et equations d'evolution lineaires stochastiques. Z. Wahrscheinlichkeits. theorie verw. Gebiete 33 (1975), 1-18.

[13] Pardoux, E.: Equations aux dérivées partielles stochastiques non lineaires monotones. These doct. sci. math. Univ. Paris Sud 1975.

[14] ZEIDLER, E.: Vorlesungen über nichtlineare Funktionalanalysis II - Monotone Operatoren --. BSB B. G. Teubner Verlagsgesellschaft: Leipzig 1977.

[15] Zeidlfr, E.: Vorlesungen über nichtlineare Funktionalanalysis [II - Variationsmethoden und Optimierung - BSB B. G. Teubner Verlagsgesellschaft: Leipzig 1978.

Manuskripteingang: 15. 12. 1982

VERFASSER:

Dr. Wilfried GreCKSCH

Sektion Mathematik der Technischen Hochschule "Carl Schorlemmer"

DDR - 4200 Merseburg, Geusaer Str. 